Proceedings of the 15th Czech and Slovak Conference on Magnetism, Košice, Slovakia, June 17-21 2013

\title{
Weak Ferromagnetism in the Helimagnet $\mathrm{Ba}_{2} \mathrm{CuGe}_{2} \mathrm{O}_{7}$
}

\author{
J. ChOVAN ${ }^{a, *}$, M. MARdeR ${ }^{b}$ AND N. PAPANICOLAOU ${ }^{c}$
}

${ }^{a}$ Department of Physics, Matej Bel University, Tajovského 40, 97401 Banská Bystrica, Slovakia

${ }^{b}$ Center for Nonlinear Dynamics and Department of Physics, The University of Texas at Austin, Austin, Texas

${ }^{c}$ Department of Physics and Institute of Plasma Physics, University of Crete, Heraklion, Greece

The Dzyaloshinskii-Moriya anisotropy, responsible for helimagnetism in $\mathrm{Ba}_{2} \mathrm{CuGe}_{2} \mathrm{O}_{7}$, has recently been shown to comprise also a weak-ferromagnetic component. We theoretically demonstrate the signatures of weakferromagnetism in the magnon spectrum when a magnetic field is applied perpendicular to the $z$ axis. We also anticipate the occurrence of unconventional domain walls in the so-called intermediate phase, and briefly elucidate the importance of the weak ferromagnetic component of the Dzyaloshinskii-Moriya anisotropy for the dynamics of driven domain walls.

DOI: $10.12693 /$ APhysPolA.126.32

PACS: 75.30.Ds, 75.30.Gw, 75.30.Kz

\section{Introduction}

$\mathrm{Ba}_{2} \mathrm{CuGe}_{2} \mathrm{O}_{7}$ is a layered spiral antiferromagnet (helimagnet) thanks to a Dzyaloshinskii-Moriya (DM) anisotropy [1]. Experiments in the late 90's [2] revealed a Dzyaloshinskii-type [3] incommensurate-tocommensurate (IC) phase transition when an external magnetic field along the $z$ axis exceeds a critical value $H_{c} \sim 2$ T. For $H<H_{c}$ the ground state (GS) is an incommensurate spiral whose period $L(H) \rightarrow \infty$ when $H \rightarrow H_{c}$. For $H>H_{c}$ the GS was thought to be a commensurate antiferromagnetic spin-flop state. Some time ago we predicted that the IC transition does not occur immediately, but is mediated by an intermediate phase [4]. In short, there exist two critical fields, $H_{c 1} \sim 1.7 \mathrm{~T}$ and $H_{c 2} \sim 2.9 \mathrm{~T}$. For $H<H_{c 1}$ the GS is a flat spiral (cycloid) that propagates along $x$ while the staggered magnetization rotates in the $x z$ plane. For $H>H_{c 1}$ the cycloid becomes a conical spiral that propagates along $x$ and nutates around the $y$ axis. Above $H_{c 2}$ the conical spiral degenerates into a spin-flop state whose staggered magnetization points along $y$. This prediction remained unexplored for almost a decade. However, new experiments have now confirmed, that the intermediate phase occurs as predicted, and that it also survives the presence of in-plane fields $H_{\perp}<0.25 \mathrm{~T}[5,6]$. Further experiments have explored the phase diagram in the presence of arbitrary canted fields [6], and observed an unexpected IC phase transition for a strictly inplane field at $H_{\perp} \sim 9 \mathrm{~T}$. Our theoretical analysis [7] suggested a nonzero weak-ferromagnetic (WF) component in the DM anisotropy, estimated its strength and explained the phase diagram. To our knowledge, $\mathrm{Ba}_{2} \mathrm{CuGe}_{2} \mathrm{O}_{7}$ is the only system where DM-induced weak ferromagnetism and helimagnetism coexist.

Our aim here is, first, to calculate the magnon spectrum in the spiral phase for the field applied in the $x y$ plane, thus providing a basis for comparison with future inelastic neutron scattering studies. Second, we predict

*corresponding author; e-mail: Jaroslav.Chovan@umb.sk

the existence of unconventional domain walls (DWs) in the intermediate phase, and elucidate the crucial role of the WF DM anisotropy for the dynamics of driven DWs.

\section{Nonlinear $\sigma$ model}

The 2D low-energy dynamics can be calculated from a nonlinear $\sigma$ model governed by the Lagrangian density, expressed in fully rationalized (dimensionless) units [4]:

$$
\begin{aligned}
\mathcal{L}= & \mathcal{L}_{0}-V ; \mathcal{L}_{0}=\frac{1}{2}\left(\partial_{0} \boldsymbol{n}\right)^{2}+\boldsymbol{h} \cdot\left(\boldsymbol{n} \times \partial_{0} \boldsymbol{n}\right) ; \\
V= & \frac{1}{2}\left(\partial_{1} \boldsymbol{n}-\boldsymbol{e}_{2} \times \boldsymbol{n}\right)^{2}+\frac{1}{2}\left(\partial_{2} \boldsymbol{n}-\boldsymbol{e}_{1} \times \boldsymbol{n}\right)^{2}+ \\
& \frac{1}{2}(\boldsymbol{n} \cdot \boldsymbol{h})^{2}+d_{z}\left(\boldsymbol{h} \times \boldsymbol{e}_{3}\right) \cdot \boldsymbol{n} .
\end{aligned}
$$

Here $e_{1}, e_{2}, e_{3}$ are unit vectors along $x, y$ and $z$; the staggered magnetization $\boldsymbol{n}=n_{1} \boldsymbol{e}_{1}+n_{2} \boldsymbol{e}_{2}+n_{3} \boldsymbol{e}_{3}$ is a unit vector field $\left(\boldsymbol{n}^{2}=1\right)$ that depends upon the inplane spatial coordinates $x, y$ as well as the time variable $t: \boldsymbol{n}=\boldsymbol{n}(x, y, t)$. Spatial and time derivatives are described by $\partial_{1}, \partial_{2}$ and $\partial_{0}$. The applied magnetic field $\boldsymbol{h}$ may point in arbitrary direction. Frequency (energy) is measured in units of $0.24 \mathrm{meV}$, magnetic field $h$ in units of $1.68 \mathrm{~T}$, and distance in units of $33.75 \AA$. The remaining free parameter $d_{z}=D_{z} / D_{\perp}$ is a dimensionless ratio of the WF DM anisotropy $D_{z}$ and its helimagnetic inplane component $D_{\perp}$. The latter has been completely suppressed in Eq. 1 except for the definitions of rationalized units quoted above. Recent analysis [7] suggested the value of $d_{z}=0.06$.

\section{Magnon spectrum for $\mathbf{H} \perp \mathbf{z}$}

Without loss of generality we assume the applied field $\boldsymbol{h}=h_{\perp} \boldsymbol{e}_{2}$. The GS is the flat spiral with $\boldsymbol{n}=n_{1}(x) \boldsymbol{e}_{1}+$ $n_{3}(x) \boldsymbol{e}_{3}$. This configuration eliminates the positive Zeeman term $(\boldsymbol{n} \cdot \boldsymbol{h})^{2}$. For $d_{z}=0$, the GS reduces to the zero-field flat spiral for any $h_{\perp}$, and the IC transition never occurs. But for $d_{z} \neq 0$, the spin spiral depends on the field through the single parameter $\bar{h}=h_{\perp} d_{z}$, and the IC transition occurs at $\bar{h}_{c}=0.3161$ [7]. The measured critical field $9 \mathrm{~T}\left(h_{\perp}=5.36\right)$ yields $d_{z}=0.06$. To obtain the magnon spectrum, we expanded Eq 1 to 2 nd order in perturbations around the GS and solved the linear system by a Bloch analysis of the type given in [4]. Our results are shown in Fig. 1. The spectrum along $Q_{1}$ has 2 modes; acoustic, depending only on $\bar{h}$, and optical, 
depending on both $\bar{h}, h_{\perp}$. When $h_{\perp} \neq 0$, the originally continuous bands split at $Q_{1}= \pm n \zeta / 2(n=1,2,3 \ldots)$ and optical modes are shifted to higher values. The lowest acoustic band is flattened and its energy is greatly reduced with rising field. At the critical field $\sim 9 \mathrm{~T}$ its energy becomes zero over the whole zone thus indicating the instability. Dispersions of the next 2 bands are also flattened and their energies become asymptotically equal to the energy of their central point at $Q_{1}=0$. Note that extra gaps at $\pm n \zeta / 2$ and instability appear only if $d_{z} \neq 0$. Along $Q_{2}$, the optical and acoustic modes are hybridized. The spectrum strongly depends on both $\bar{h}$, $h_{\perp}$. The lowest band is quadratic at $h_{\perp}=0$ but becomes linear for strong fields. The flat spiral does not "see" the instability along $Q_{2}$.
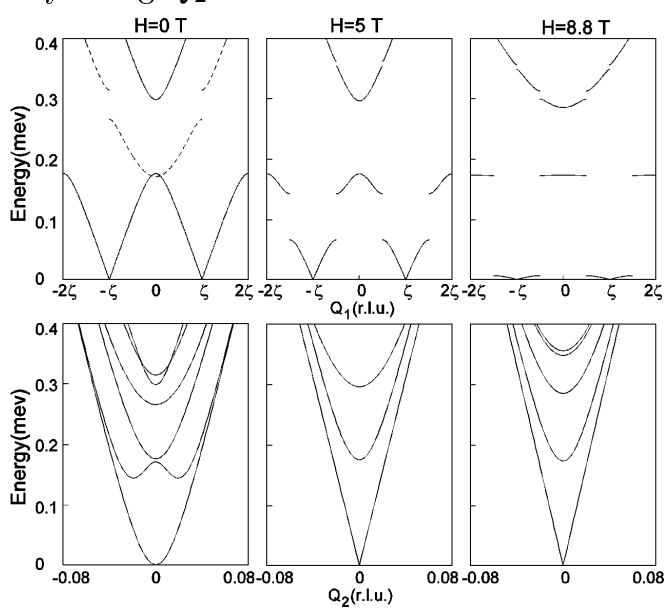

Fig. 1. Magnon spectrum in a magnetic field applied perpendicular to the $z$ axis. Acoustic and optical modes in the spectrum along $Q_{1}$ are distinguished by solid and dashed lines. The wave numbers $Q_{1}, Q_{2}$ are measured in relative lattice units defined in Ref. [4].

\section{Domain walls in the intermediate phase}

For $\boldsymbol{H}=H_{z} \boldsymbol{e}_{3}$ and $H_{z}<1.7 \mathrm{~T}$, the GS is the flat spiral that propagates along $x$ and rotates in the $x z$ plane. The GS is nondegenerate, with chirality $\chi=$ $+1\left(\chi \equiv \operatorname{sgn}\left[\boldsymbol{e}_{2} \cdot\left(\boldsymbol{n} \times \partial_{1} \boldsymbol{n}\right)\right]\right)$ due to the helimagnetic part of the DM anisotropy. The degeneracy with respect to the $U(1)$ symmetry $[4,7]$ is not crucial and is disregarded here. Stable DW are not possible in this regime. In the intermediate phase $\left(1.7 \mathrm{~T}<H_{z}<2.9 \mathrm{~T}\right)$, chirality is again $\chi=+1$, but the GS conical spiral $\boldsymbol{n}=\left[n_{1}(x) \boldsymbol{e}_{1}+n_{3}(x) \boldsymbol{e}_{3}\right]+\kappa n_{2}(x) \boldsymbol{e}_{2}, \kappa= \pm 1$, is doubly degenerate with respect to "conicity" $\kappa$, see Fig. $2 \mathrm{a}$,b. Here, DWs may exist as truly stable localized nonlinear excitations connecting two topologically distinct spatialy modulated vacua. An example is in Fig. 2c,d, with DW obtained by a direct numerical minimization of the potential $V$ of Eq. 1 for the prototype DW. This type of DWs in DM helimagnets is novel and differs significantly from its more conventional counterparts. For example, DWs discussed here possess only discrete translational invariance. Note that the DW depends on the GS, whose properties, such as magnitude of $n_{2}$ and the spiral period L, can be tuned by changing $H_{z}$. Finally, when the field acquires a nonzero transverse component $H_{\perp}$ applied along $x\left(\boldsymbol{H}=H_{\perp} \boldsymbol{e}_{1}+H_{z} \boldsymbol{e}_{3}\right)$, the WF DM anisotropy lifts the degeneracy of the 2 GS through the term $d_{z}\left(\boldsymbol{h} \times \boldsymbol{e}_{3}\right) \cdot \boldsymbol{n}=-d_{z} h_{\perp} n_{2}$ in the potential $V$ of Eq (1).

Thus, $H_{\perp}$ may trigger the dynamics of DWs via mechanism similar to that in ordinary weak ferromagnets [8].
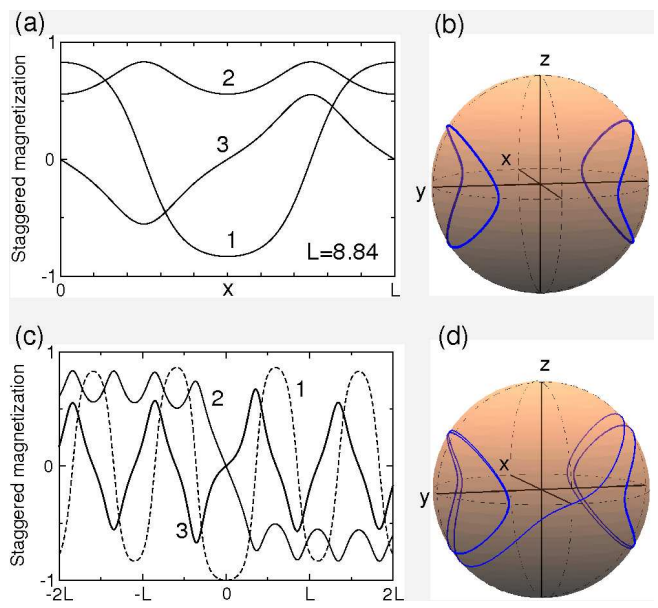

(d)

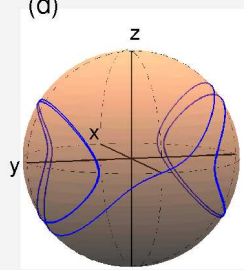

Fig. 2. GS and DW spin configurations calculated for $H=2.05 \mathrm{~T}$ applied along $z$. (a) The components of the GS conical spin spiral with period $L$ along $x$. (b) The two degenerate GS $\boldsymbol{n}=\left(n_{1}, \pm n_{2}, n_{3}\right)$. The thick lines on the sphere are traced by the endpoint of $\boldsymbol{n}$ along $x$ during one period $L$. (c) A portion of the DW profile around the centre $x=0$. At $x \rightarrow \pm \infty$ the actual solutions correspond to the two degenerate GS. (d) Path traced by the endpoint of $\boldsymbol{n}$ for the DW solution.

\section{Conclusions}

We have shown that weak ferromagnetism in $\mathrm{Ba}_{2} \mathrm{CuGe}_{2} \mathrm{O}_{7}$ becomes manifested in the magnon spectrum when the magnetic field is applied in the $x y$ plane. We have also shown that unconventional domain walls may exist in the intermediate phase of the system.

\section{Acknowledgments}

J.C. acknowledges the support by the Slovak Research and Development Agency through APVV-0027-11.

\section{References}

[1] I.E. Dzyaloshinskii, Sov.Phys. JETP 5, 1259 (1957); T. Moriya, Phys. Rev. Lett. 4, 228 (1960).

[2] A. Zheludev, S. Maslov, G. Shirane, Y. Sasago, N. Koide, K. Uchinokura, Phys. Rev. Lett. 78 , 4857 (1997).

[3] I.E. Dzyaloshinskii, Sov.Phys. JETP 20, 665 (1965).

[4] J. Chovan, N. Papanicolaou, S. Komineas, Phys. Rev. B 65, 064433 (2002).

[5] S. Mühlbauer, S. Gvasaliya, E. Pomjakushina, and A. Zheludev, Phys. Rev. B 84, 180406(R) (2011).

[6] S. Mühlbauer, S. Gvasaliya, E. Ressouche, E. Pomjakushina, A. Zheludev, Phys. Rev. B 86, 024417 (2012).

[7] J. Chovan, M. Marder, N. Papanicolaou, Phys. Rev. B 88, 064421 (2013).

[8] N. Papanicolaou, Phys. Rev. B 55, 12290 (1997). 